\title{
Performance Evaluation of Packet Delivery Ratio for Mobile Ad-hoc Networks
}

\author{
K.Prabha \\ Periyar Univerity PG Extension Centre \\ Dharmapuri,Tamilnadu \\ India
}

\author{
R.Anbumani \\ Periyar Univerity PG Extension Centre \\ Dharmapuri,Tamilnadu \\ India
}

\begin{abstract}
Protocols are used to maintain data integrity, delivery, throughput and packet drop ratio in mobile ad-hoc network. It is most important to study performance metrics factors like throughput and packet drop ratio of proactive and reactive protocols in mobile ad-hoc network. In this paper, a comparative performance analysis is based on protocols like the Dynamic Source Routing, the Ad-hoc On-demand Distance Vector, the Destination Sequenced Distance Vector and the Optimized Link State Routing protocols using NS2 simulator.
\end{abstract}

Keywords: Ad-hoc routing protocols, Throughput, Packet Delivery Ratio, AODV, DSDV, DSR, OLSR.

\section{INTRODUCTION}

A Mobile Ad-hoc networking is an emerging technology that allows each node can connect by wireless communication links, without any base station [8]. Mobile Ad-hoc networking have several characteristics bandwidth, energy and physical security are limited and topology dynamics. Therefore the routing protocols used in wired network are not suited for mobile Ad-hoc networking. Many routing protocols have been proposed

for mobile Ad-hoc networking can be classification as reactive and proactive protocols [3]. In Reactive are only discovered when they are actually needed. In contrast, in proactive routing each node continuously maintain route between pair of nodes. In this paper focused on Ad-hoc On-demand Distance Vector and Dynamic Source Routing as reactive protocol and Destination Sequenced Distance Vector and Optimized Link State Routing as proactive protocol. Ad-hoc On-demand Distance Vector is an on demand routing algorithm. When a node needs to send data to a specific destination it creates a Route Request and broadcast. Next nodes create a reverse route for itself for destination. When the request reaches a destination

Dr. K. Prabha, Assistant Professor, Department of computer science, Periyar University PG Extension Centre, Dharmapuri - 636705, INDIA node it creates again a Reply which contains the number of hops that are require to reach the destination.

All nodes forwarding this reply to the source node create a forward route to destination [3].MANETS are Multi-Hop wireless networks since one node may not be indirect communication range of other node. Ad hoc networks are viewed to be suitable for all situations in which a temporary communication is desired. The technology was initially developed keeping in mind the military.Applicationssuch as battle field in an unknown territory where an infrastructurenetwork is almost impossible to have or maintain.A Mobile Ad hoc network is a collection of wireless mobile nodes where nodes come together by forwarding packets andalso exchange information over direct wireless range.

The routing protocol such as ADOV, DSR, DSDV and OLSR have been investigated on the MANETs in the past few years. The investigation of the performance of these protocols on the MANETs has produced many useful results. The power constrained is one of the main design constraints in MANET and all effort is to be channel towards reducing power. Moreover network generation is a key design metric in MANETs. Since every node has to perform the functions of a router, if some nodes pass away early due to lack of energy and it will not be probable for other nodes to communicate with each other. Hence the network will get disjointed and the network lifetime will be unfavorably affected. It has the lifetime of prediction routing protocol for MANETs that maximizes the network lifetime with sentence routing solutions that minimize the inconsistency of the remaining energies of the nodes in the network.

Dynamic Source Routing is a reactive protocol as Adhoc On-demand Distance Vector protocol. Difference in Adhoc On-demand Distance Vector and Dynamic Source Routing (phone: 04342-230399; e-mail: prabhaeac@gmail.com). R.Anbumani, Ph.D Research scholar, Department of computer science, Periyar University PG Extension Centre, Dharmapuri 636705,TAMILNADU,INDIA. 
is that Ad-hoc On-demand Distance Vector only stores address of next node to the destination but Dynamic Source Routing stores complete path from source to destination including all the intermediate nodes. Source of the packet discovers the route through which to forward the packets. Sender carries in data packet header the complete ordered list of nodes through which the packet must pass [4][2].Destination Sequenced Distance Vector It is a table-driven routing scheme for Ad-hoc mobilenetworks based on the Bellman-Ford algorithm [6].

Routing table contains the sequence number assigned by destination node. The sequence number is used to avoid loop formation and distinguish stale routes from new ones.

The stations periodically transmit their routing tables to their immediate neighbors. The routing table updates can be sent in two ways: a "full dump" or an "incremental" update.The Optimized Link State Routing is a table driven, proactive routing protocol developed for Mobile Ad-hoc networks. Optimized Link State Routing uses the concept of Multi point Relays to reduce the effect of flooding messages to all nodes in the network, Optimized Link State Routing selects a subset of nodes to be part of a relaying backbone. Optimized Link State Routing works with a periodic exchange of messages like Hello messages and Topology Control message only through its Multi point Relays. So, contrary to classic link state algorithm, instead of all links, only small subsets of links are declared.

This paper involves study of four routing protocols (Ad-hoc On DemandDistance Vector Routing, Optimized Link State Routing, Dynamic Source Routing and Distance SequencedDistance Vector), and performance comparisons between these routing protocols on the basis of performance metrics throughput, packet delivery ratio, Packet dropped, jitter and end to end delay.A mobile ad hoc network (MANET) is a group of communications and wireless nodes which cooperatively and spontaneously at any infrastructure from base station and access points through administration. Every node can communicate to each other at directly with all dynamically multi-hop route.

\section{RELATED WORK}

\section{A. Packet Delivery Ratio}

It is the ratio of actual packet delivered to total packets sent. The following table shows the values of the various parameters used during simulation of these protocols.

\section{B. Mobile Ad-hoc Networking}

Mobile Ad hoc Networking (MANET) is a group of independent network mobile devices that are connected over various wireless links. It is relatively working on a constrained bandwidth. The network topologies are dynamic and may vary from time to time. Each device must act as a router for transferring any traffic among each other. This network can operate by itself or incorporate into large area network (LAN). There are three types of MANET. It includes Vehicular Ad hoc Networks (VANETs), Intelligent Vehicular Ad hoc Networks (In VANETs) and Internet Based Mobile Ad hock Networks (I MANET).

\section{Reactive (Source-Initiated On-Demand Driven)}

These protocols try to eliminate the conventional routing tables and consequently reduce the need for updating these tables to track changes in the network topology. When a source requires to a destination, it has to establish a route by route discovery procedure, maintain it by some form of route maintenance procedure until either the route is no longer desired or it becomes inaccessible, and finally tear down it by route deletion procedure. In pro-active routing protocols, routes are always available (regardless of need), with the consumption of signaling traffic and power. Some of reactive routing protocols are Ad hoc On-Demand Distance Vector (AODV), Dynamic Source Routing (DSR).

\section{Hybrid protocols}

Hybrid protocols combine the features of reactive and proactive protocols. These protocols have the advantage of both proactive and reactive routing protocols to balance the delay which was the disadvantage of Table driven protocols and control overhead (in terms of control packages). Main feature of Hybrid Routing protocol is that the routing is proactive for short distances and reactive for long distances. The common disadvantage of hybrid routing protocols is that the nodes have to maintain high level topological information which leads to more memory and power consumption. Examples: ZRP (Zone Routing Protocol).

\section{Mobile Ad Hoc Sensor NeTwork}

A mobile ad-hoc sensor network follows a broader sequence of operational, and needs a less complex setup procedure compared to typical sensor networks, which communicate directly with the centralized controller. A mobile ad-hoc sensor or Hybrid Ad Hoc Network includes a number of sensor spreads in a large geographical area. Each sensor is proficient in handling mobile communication and has some level of intelligence to process signals and to transmit data. In order to support routed communications between two mobile nodes, the routing protocol determines the node connectivity and routes packets accordingly. This condition has makes a mobile ad-hoc sensor network highly flexible so that it can be deployed in almost all environments.

The Traffic Types in the Ad Hoc Networks are so different from the infrastructure wireless network, and then now we will see these types. The first one Peer to Peer (P2P) the second remote to remote and lastly dynamic traffic. So now we will discuss every one [19]. Firstly, Peer to peer: communication between two nodes in the same area, that means which are within one hop. Network traffic (in bits per 
second) is usually fixed. Secondly, remote to remote: Communication between two nodes beyond a single hop, but maintain a stable route between them. This may be the result of a number of Nodes, to stay within the range of each other in one area or may move as a group. Movement it's a similar to the standard network traffic. Finally, Dynamic traffic: its will happened when the nodes are movie dynamically around and then the routers must be reconstructed. This results in a poor connectivity and network activity in short bursts. For example in IEEE 802.11 network and the basic structure divided into two types firstly infrastructures wireless LAN, the second structure Ad Hoc Wireless LAN.

\section{Pursue Mobility Model}

The Pursue Mobility Model attempts to represent MNs tracking a particular target. For example, this model could represent police officers attempting to catch an escaped criminal. The Pursue Mobility Model consists of a single update equation for the new position of each MN. Where acceleration(target - old position) is information on the movement of the MN being pursued and random vector is a random offset for each $\mathrm{MN}$.

Table 1: Simulation Parameters

\begin{tabular}{|c|c|}
\hline Parameter & Value \\
\hline Routing protocols & $\begin{array}{c}\text { AODV, DSDV, DSR, } \\
\text { OLSR }\end{array}$ \\
\hline No. of Mobile Nodes & 25,50 \\
\hline Simulation Period (s) & 150 \\
\hline MAC type & 802.11 \\
\hline Avg speed (m/s) & 11.40 \\
\hline Pause Time (s) & $0,10,20,30,40,50$ \\
\hline Simulation area & $500 * 500$ \\
\hline
\end{tabular}

\section{PERFORMANCE EVALUATION}

\section{A. Effect of varying number of nodes}

The results of speeds ratios varying density of nodes within the network area node speed correlation. Simulation results on MAT Lab exhibit the effect of the MNs population on the mobility rate.

It is considered that all mobile nodes are prepared with IEEE 802.11 network interface card, with data rates of $2 \mathrm{Mbps}$.
Arbitrary connections were created using CBR traffic such that everyone node has chance to attach to every other node. Packet size was 512 bytes. The primary battery ability of every node is 100 units. Simulation parameters taken in the performance evaluation of NMDC campaigns are listed.

\section{B. Packet Delivery Ratio}

The packet delivery ratio is the ratio of the number of packets received by the destination to the number of packets generated by the source node. The Proposed system performs the best in terms of packet delivery ratio followed by AODV.This is because the established route by proposed protocol are stayed alive longer time compared to that of other protocols and stable in nature. Hence, the numbers of packets dropped are lesser due to lack of energy at intermediate node of the route between source and destination. In contrary to AODV where packets may get dropped due to link failures which may occur for insufficient energy of nodes in an established route.

\section{EXPERIMENTAL RESULTS}

Table 1: PDR for 25 nodes

\begin{tabular}{|c|c|c|c|}
\hline $\begin{array}{c}\text { Pause } \\
\text { time }\end{array}$ & AODV & DSDV & DSR \\
\hline $\mathbf{0}$ & 99.85 & 97.78 & $\mathbf{1 0 0}$ \\
\hline $\mathbf{1 0}$ & 99 & 88.48 & $\mathbf{9 8 . 7 7}$ \\
\hline $\mathbf{2 0}$ & 99.46 & 76.08 & $\mathbf{9 9 . 5 6}$ \\
\hline $\mathbf{3 0}$ & 99.55 & 89.58 & $\mathbf{9 9 . 0 1}$ \\
\hline $\mathbf{4 0}$ & 98.76 & 74.68 & $\mathbf{9 9 . 0 2}$ \\
\hline \hline $\mathbf{5 0}$ & $\mathbf{9 9 . 1 6}$ & $\mathbf{8 2 . 8}$ & $\mathbf{9 9 . 9 5}$ \\
\hline
\end{tabular}




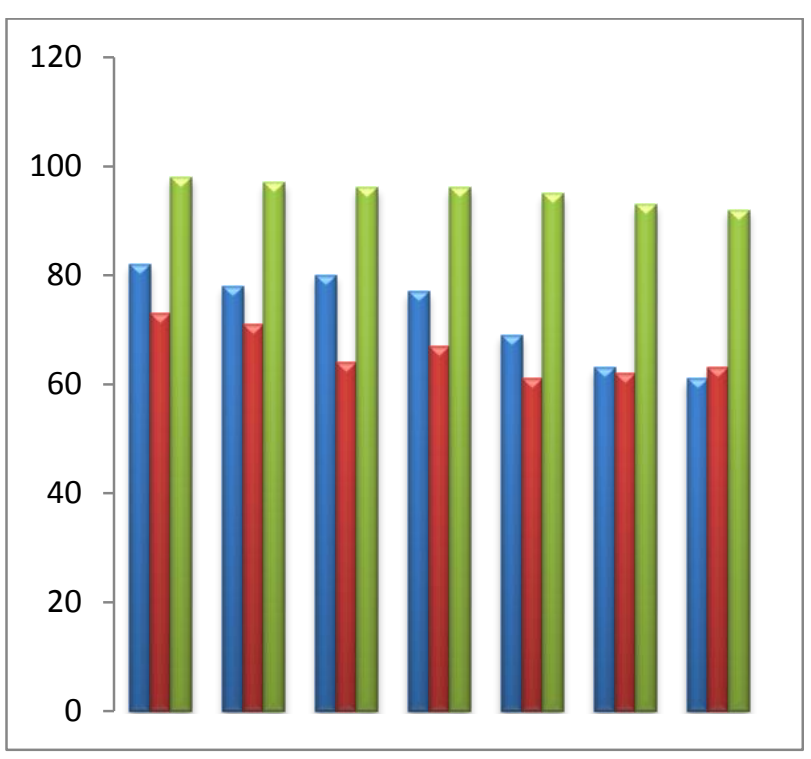

Figure: 1 PDR for 25 nodes

The node lifetime is a declared as the level of density classifier and node mobility models. Node Lifetime refers to the unpredicted loss of life in the nodes. In MANET, the nodes lifetime increases when slow, medium, height mobility state.

The number of the nodes increases, the speed of the nodes decreases. This is because nodes are closed to each other making mobility difficult. One may say that the network under study is crowded with nodes. As the number of nodes increases it could be better to increase the speed of the nodes, so that the nodes can move fast to give room to other nodes. In all, if the number of nodes is higher then speed must be increased for better mobility which is the opposite in the case of fewer nodes. It also means that nodes have a number of hops to get to their destination nodes. The larger the number of nodes means it require higher speed in order to get to a particular location.

A decreased in the number of nodes in an area implies a decreased in the connectivity of nodes i.e., each node has fewer neighbours. A decreased in connectivity also implies lesser information exchange hence less input to the algorithm. An increased in the number of nodes implies high connectivity among nodes; more information is exchanged and hence more input to the algorithm. It is therefore important to conclude that when the nodes are many in a particular location, it would be wise to increase the speed to a certain limit.

\section{CONCLUSION}

The number of mobile nodes the speeds of the MNs and their distribution in a location. It may claim that as the number of MNs increased the speeds of MNs may also fall but to a certain limit. It was therefore necessary to increase the speed MNs to give room to other nodes or make it possible for free movement.
The performance of Ad-hoc On demand Distance Vector, Destination Sequenced Distance Vector, and Dynamic Source Routing \& results Optimized Link State Routing on the basis metrics like of throughput and packet delivery ratio. These analyses were made while varying the value of pause time parameter. As per the analysis, the throughput results Optimized Link State Routing were the best for both cases of number of nodes. Hence they performed better than reactive protocols in these respects. These protocols show consistency in their throughput values, especially Optimized Link State Routing, which was rarely effected by changes in pause time or number of nodes. Another observation that can be made on the basis of these simulation data is that the maximum effect of change in pause time was seen on Destination Sequenced Distance Vector. The value for its metrics Packet Delivery Ratio and throughput showed deep variations as compared to other protocols.

\section{REFERENCES}

[1] Li Layuan, Li Chunlin and YaunPeiyan, "Performance evaluation and simulations of routing protocols in ad hoc networks", Computer Communications, Elsevier, vol.30, pp.1890-1898, 2007.

[2] AzzedineBoukerche, "Performance Evaluation of Routing Protocols for Ad Hoc Wireless Networks", Mobile Networks and Applications, vol.9, pp. 333-342, 2004.

[3] S. Mohapatra and P.Kanungo, "Performance analysis of AODV, DSR, OLSR and DSDV Routing Protocols using NS2 Simulator", in proc. International Conference on Communication Technology and System Design, pp. 69-76, 2011.

[4] AsmaTuteja, Rajneesh Gujral and Sunil Thalia, "Comparative Performance Analysis of DSDV, AODV and DSR Routing Protocols in MANET using NS2", in proc. International Conference on Advances in Computer Engineering, pp. 330- 334,2010.

[5] M. Shobana and Dr. S. Karthik, "A Performance Analysis and Comparison of various Routing Protocols in MANET", in proc. International Conference on Pattern Recognition, Informatics and Mobile Engineering (PRIME), pp 391-392, 2013.

[6] Gurpinder Singh and Jaswinder Singh "MANET: Issues and Behavior Analysis of Routing Protocols", International Journal of Advanced Research in Computer Science and Software Engineering, vol. 2, Issue 4, pp. 219-227, 2012.

[7] K.RameshReddy,S. VenkataRaju and N.Venkatadri, "Reactive, Proactive MANET Routing Protocol Comparison", International Journal of Video\& Image Processing and Network Security, vol.12, pp. 22-27,2012.

[8] Tamilarasan-Santhamurthy, "A Comparative Study of Multi-Hop Wireless Ad-Hoc Network Routing Protocols in MANET", IJCSI International Journal of Computer Science Issues, vol. 8, Issue 5,

[9]pp. 176-184, 2011.M. Xuan Shi and Kai Liu, “A contentionbased beaconless geographic routing protocol for mobile ad hoc networks", International Conference on Communications and Networking, pp.840-843, Aug 2008. 
[10]MajidKhabbazian, Ian F. Blake, and Vijay K. Bhargava, "Local Broadcast Algorithms in Wireless Ad Hoc Networks: Reducing the Number of Transmissions", IEEE Transactions on Mobile Computing, Vol.11, No.3, March 2012.

[11]V. Davies. "Evaluating Mobility Models within an Ad hoc Network", Master's Thesis, Colorado School of Mines, 2000.

[12]Gunnar Karisson et al., "A Mobility Model for Pedestrian Content Distribution”, SIMUTools '09 workshops, March 2-6, 2009, Rome Italy.

[13]J.C. Cano, P. Manzoni, and M. Sanchez."Evaluating the Impact of Group Mobility on the Performance of Mobile Ad Hoc Networks".In Proc. IEEE ICC, 2004.

[14]K. Wang and B. Li, "Group Mobility and Partition Prediction in Wireless Ad-hoc Networks", IEEE International Conference on Communications, ICC 2002, New York, April 2002.

[15]Indrani Das, D.K Lobiyal and C.P Katti Multipath Routing Protocol in MANET", Published in the proceeding of fourth International Coference on Advances in Computing and Information Technology ACITY 2014 . 